\title{
Development of a generic method for the determination of proton- pump inhibitors by capillary zoneelectrophoresis
}

\author{
Lajos Attila Papp${ }^{1}$, Árpád Gyéresi', Gabriel Hancu*,1, Eleonora Mircia' ${ }^{1}$, Hajnal Kelemen ${ }^{1}$ \\ ${ }^{I}$ Department of Pharmaceutical Chemistry, Faculty of Pharmacy, University of Medicine and Pharmacy, Tîrgu Mureş, \\ Romania
}

\begin{abstract}
A generic capillary zone electrophoresis method was developed for the analysis of four proton pump inhibitors: omeprazole, pantoprazole, lansoprazole and rabeprazole. During preliminary analysis screening of phosphate buffers at different $\mathrm{pH}$ levels was performed, in order to determine the optimum $\mathrm{pH}$ domain suitable for the simultaneous determination of all studied compounds. A face centered central composite design was employed for the optimization of separation conditions. The effect of buffer concentration, $\mathrm{pH}$ and applied voltage was studied; resolution between peaks and migration time of the last compound were considered as responses. Other factors as system temperature, injection parameters, capillary length, were held constant during the optimization process. The optimized conditions consisted of $40 \mathrm{mM}$ phosphate background electrolyte at $\mathrm{pH} 5.0,+25 \mathrm{kV}$ applied voltage and $20^{\circ} \mathrm{C}$ temperature. The migration order of the analytes was as follows: rabeprazole, omeprazole, lansoprazole and pantoprazole. Full resolution of all analytes was achieved within 9 minutes. The method was validated and proved to be suitable in terms of repeatability, sensitivity, linearity, accuracy and robustness. Determinations from commercially available pharmaceutical formulation were performed for omeprazole; good reproducibility and recovery were obtained.
\end{abstract}

Keywords: Capillary electrophoresis. Proton-pump inhibitors. Experimental design.

\section{INTRODUCTION}

Proton pump inhibitors (PPIs) are currently the most effective and frequently used agents in acid suppression therapy. Their indications include disorders like dyspepsia, peptic ulcer, gastroesophageal reflux disease, ZollingerEllison syndrome and eradication of Helicobacter pylori. PPIs act by blocking irreversibly the so called proton pump (hydrogen/potassium adenosine triphosphatase enzyme) localized in the luminal membrane of gastric parietal cells, producing an antisecretory effect for at least 24 hours (Strand, Kim, Peura, 2017).

The classical members of the PPIs, including omeprazole, pantoprazole, lansoprazole and rabeprazole are pyridinyl-methyl-sulfinyl-benzimidazole derivatives with a sulfoxide group attached at position 2 of the benzimidazole moiety. All of these compounds are weak bases having nearly equal pKa values around 4 (from 3.83

*Correspondence: G. Hancu. Faculty of Pharmacy, University of Medicine and Pharmacy from Tîrgu Mureș, Gh. Marinescu 38, 540139 TîrguMureș, Romania. Tel: 0040-265-215551.E-mail: gabriel.hancu@umftgm.ro iD for pantoprazole to 4.53 for rabeprazole) (Strand, Kim, Peura, 2017).

The chemical structures of the studied PPIs are presented in Figure 1.

In recent years, capillary electrophoresis (CE) has developed as an easily operable, cost-effective and an environmental friendly technique where the separation is based on the differences between the electrophoretic mobilities of the analytes in an electric field. Nowadays, CE represents an interesting alternative to high performance liquid chromatography (HPLC) methods in the analysis of pharmaceuticals (Deeb et al., 2014).

Recently, statistical experimental designs have been increasingly employed as a useful option in the development of analytical methods. The use of experimental design approaches enables the identification of significant analytical factors and interaction effects, and can help in establishing precisely the optimal combination of the experimental factors. An experimental design method is equipped to address several major experimental concerns for which the traditional "one factor ata time" (OFAAT) trial and error development 
<smiles>COc1ccc2[nH]c([SH](C)(=O)Cc3ncc(C)c(OC)c3C)nc2c1</smiles><smiles>Cc1c(OCC(F)(F)F)ccnc1CS(=O)[S@](C)(=O)c1nc2ccccc2[nH]1</smiles><smiles>COc1cnc(CS(=O)(=O)c2nc3cc(OC(F)(F)F)ccc3[nH]2)cc1OC</smiles><smiles>[R20][R6]O[Na]</smiles>

FIGURE 1 - Chemical structures of the studied PPIs (* denotes the chiral centers).

technique is not suited (Orlandini, Gotti, Furlanetto, 2014).

Experimental design methodologies used in analytical chemistry can be classified in screening designs (fractional factorial, Plackett-Burman etc.) and optimization designs (Central composite, Doelhert etc.). Central composite designs (CCDs) allow the estimation of linear, interaction and quadratic effects of the studied factors on the analytical response(s). Face centered central composite design is the simplest way to carry out a CCD, since each factor of interest has three experimental levels, by maintaining the design capability to use nonlinear models (Dejaegher, Mangelings, Vander Heyden, 2013).

In the last two decades several CE methods have been published for the analysis of PPIs (El-Kommos et al., 2015). A non-aqueous CE method has been reported for purity analysis of five different pyridinylmethylsulfinyl-benzimidazole derivatives; $N$-methylformamide was found to have the best properties in respect of both electrophoretic behavior and high solubility of the model compounds (Tivesten et al., 1999). The determination of lansoprazole from capsules and enteric coated pellets have been achieved by CE using a $20 \mathrm{mM}$ borate buffer at $\mathrm{pH} 8.7$ as background electrolyte (DogrukolAk, Tuncel, Aboul-Enein, 2001). In another study a $10 \mathrm{mM}$ sodium tetraborate buffer at $\mathrm{pH} 9.0$ have been used for the determination of rabeprazole (Garcia et al., 2005). Simultaneous determination of omeprazole and lansoprazole has been achieved also using $50 \mathrm{mM}$ phosphate buffer at pH 9.0 (Lin, $\mathrm{Wu}, 2005$ ). However, to our knowledge no generic CE method for the simultaneous determination of all four PPIs currently used in therapy has been published so far.

$\mathrm{CE}$ has been especially used successfully for the chiral analysis of PPIs. A validated CE method have been published for the chiral separation of three PPIs (pantoprazole, omeprazole and lansoprazole) using bovine serum albumin as chiral selector, in a phosphate buffer at $\mathrm{pH}$ 7.4; but because of strong absorption of the buffer system, the limits of detection were rather poor (Eberle, Hummel, Kuhn, 1997). Better results were obtained when using CD derivatives as chiral selectors. The use of $\beta-\mathrm{CD}$ as chiral selector in phosphate buffer at $\mathrm{pH} 4.0$ has been investigated for the determination of omeprazole enantiomers from tablets; the results obtained were compared with the results obtained with a HPLC method (Bonato, Paias, 2004). The enantiomeric separation of omeprazole has been achieved also using methyl- $\beta-C D$, in strong acidic phosphate buffer at $\mathrm{pH} 2.2$ (Nevado, Penalvo, Dorado, 2005). The enantiomers of omeprazole and its metabolite 5-hydroxyomeprazole have been separated by non-aqueous $\mathrm{CE}$ with heptakis-(2,3-di- $O$-methyl$6-O$-sulfo)- $\beta$-CD as chiral selector in an ammonium acetate buffer acidified with formic acid in methanol (Olsson et al., 2006). The chiral analysis of omeprazole and 5-hydroxyomeprazole, using aqueous CE and highly sufated- $\beta-C D$ as chiral selector, with UV and MS detection have been also reported (Olsson, Marlin, Blomberg, 2007). A validated method for combined analysis of the enantiomeric purity of both esomeprazole and related substances was based on the use of Tris-phosphate buffer and 2-hydroxypropyl- $\beta-C D$ as chiral selector (Estevez et al. 2014). Chiral separation of pantoprazole has been achieved by CE using a mixed borate-phosphate buffer at pH 6.50 with sulfated- $\beta-C D$ as chiral selector (Guan et al., 2012). A method suitable for enantiomeric separation of pantoprazole, lansoprazole, omeprazole and tenatoprazole using a copper(II)-1-histidine complex and HP- $\beta$-CD as dual chiral selector system in a phosphate buffer have been also reported (Guan et al., 2014). An ephedrinebased chiral ionic liquid, served as both chiral selector and 
background electrolyte in an innovative non-aqueous $\mathrm{CE}$ system used for the enantioseparation of rabeprazole and omeprazole; ion-pair interaction and hydrogen bonding may be responsible for the main separation mechanism (Ma et al., 2010). A validated CE method has been reported for the quantitative determination of lansoprazole enantiomers from pharmaceutical formulations using phosphate buffer at $\mathrm{pH} 2.2$, and $\beta-\mathrm{CD}$ as chiral selector (Nevado et al., 2009). A study regarding the enantiomeric separation of omeprazole and pantoprazole have been also published by our research group; the BGE consisted in a phosphate buffer at $\mathrm{pH} 2.5$ and randomly methylated $\beta-\mathrm{CD}$ as chiral selector for omeprazole enantioseparation, and phosphate buffer at $\mathrm{pH} 7.0$ and sulfobutyl ether- $\beta-\mathrm{CD}$ for the enantioseparation of pantoprazole, respectively (Hancu, Papp, Rusu, 2015).

The aim of this work was to develop a new simple, rapid and precise generic method for the determination of four PPIs (omeprazole, lansoprazole, rabeprazole and pantoprazole); to optimize the analytical conditions of the separation by an experimental design approach, and to evaluate the analytical performance of the developed method.

\section{MATERIAL AND METHODS}

\section{Apparatus}

Experiments were performed with an Agilent 1600 CE system (Waldbronn, Germany) equipped with a diodearray detector. Separations were performed using $48 \mathrm{~cm}$ length (effective length $40 \mathrm{~cm}) \times 50 \mu \mathrm{m}$ I.D. uncoated fused-silica capillaries (Agilent). Electropherograms were recorded and processed by Chemstation 7.01 software (Agilent). Buffer solution $\mathrm{pH}$ was determined with a Terminal $740 \mathrm{pH}$-meter (Inolab, Germany). Design-Expert 7.0 statistical software (Stat-Ease, Minneapolis, USA) was used for experimental design method optimization. Microsoft Excel 2010 was used for statistical analysis during method validation.

\section{Chemicals and reagents}

Omeprazole was obtained from Alfa Aesar (Karlsruhe, Germany) while pantoprazole sodium from Cipla (Mumbai, India), both substances were of pharmaceutical grade; lansoprazole and rabeprazole sodium were USP reference standards (Rockville, USA). The following reagents of analytical grade were used: phosphoric acid (Chimopar, Bucharest, Romania), methanol, sodium hydroxide (LachNer, Neratovice,
Czech Republic), disodium hydrogen phosphate, sodium dihydrogen phosphate and sodium metabisulfite (Merck, Darmstadt, Germany). Purified water was obtained using a Milli-Q Plus water-purification system (Millipore, Milford, USA). For determinations from commercially available pharmaceutical formulation Omez capsules (Dr Reddy's, India) were used, each capsule containing 20 mg omeprazole.

\section{Sample preparation}

Sample stock solutions were prepared by dissolving the analytes in methanol at a concentration of $1 \mathrm{mg} \mathrm{mL}^{-1}$; and subsequently diluted with the same solvent to appropriate concentrations. All buffers and sample solutions were filtered through $0.45 \mu \mathrm{m}$ syringe filters and degassed for 5 minutes before use. The samples were introduced at the anodic end of the capillary by hydrodynamic injection, the detection taking place at the cathodic end.

For the analysis of commercial formulation the contents of ten capsules were mixed and powdered and an amount of powder equivalent to the weight of one capsule was dissolved in $100 \mathrm{~mL}$ methanol by sonication for $10 \mathrm{~min}$. The solution was filtered through a $0.45 \mu \mathrm{m}$ syringe filter, and diluted with methanol to the appropriate concentrations.

\section{Electrophoretic conditions}

Before first use, capillaries were washed for 30 minutes with $0.1 \mathrm{M}$ sodium hydroxide solution and with water for 15 minutes followed by conditioning for 30 minutes with the background electrolyte (BGE) used in the analysis. Between runs the capillaries were rinsed for 2 minutes with $0.1 \mathrm{M}$ sodium hydroxide, then for 1 minute with water and finally for 2 minutes with the BGE. In the preliminary analysis we used "standard" electrophoretic conditions for a $\mathrm{CE}$ analysis: temperature $20^{\circ} \mathrm{C}$, applied voltage $+20 \mathrm{kV}$, injection pressure/time $50 \mathrm{mbar} / 2 \mathrm{sec}$.,

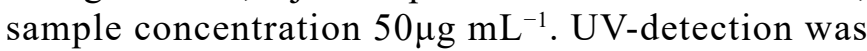
applied, detection wavelengths were set to 210, 240 and $300 \mathrm{~nm}$, taking into consideration the absorption maxima of the analytes.

\section{RESULTS AND DISCUSSION}

\section{Preliminary analysis}

Taking into consideration the fact that the simultaneous determination of all four studied 
compounds demonstrates the capability of the method for the determination of these compounds possessing similar structural properties, conditions suitable for the simultaneous separation of all four analytes were searched. Preliminary experiments were conducted using phosphate buffers at different $\mathrm{pH}$ levels in the range of 2.5-9.0. The buffer concentration in the initial experiments was set to $25 \mathrm{mM}$.

All PPIs have low stability in acidic condition; therefore some authors recommend the use of an antioxidant agent to prevent PPIs degradation (Bonato, Paias, 2004; Estevez et al., 2014). For this purpose $5 \mathrm{mM}$ sodium metabisulfite was added to acidic BGEs; this measure proved to be efficient in preventing analyte degradation during the electrophoretic procedures.

The $\mathrm{pH}$ of the BGE plays a major role in the selectivity of separation influencing not only the magnitude of the electroosmotic flow (EOF) but also the ionization grade of the analytes. Therefore the selection of an adequate $\mathrm{pH}$ domain can have a crucial effect on the simultaneous separation of analytes with similar physicochemical and structural properties.

Although all PPIs could be detected throughout the studied $\mathrm{pH}$ range ( $\mathrm{pH}$ 2.5-9.0), simultaneous separation was achieved only when using phosphate buffer at $\mathrm{pH}$ 5.0. The migration order was the following: rabeprazole, omeprazole, lansoprazole and pantoprazole. The first two and the last two peaks have been baseline separated with high resolution values; but the resolution between omeprazole and lansoprazole was not satisfying (1.16), while the analysis time was above 10 minutes. The electropherogram of the separation under initial electrophoretic conditions is presented in Figure 2.

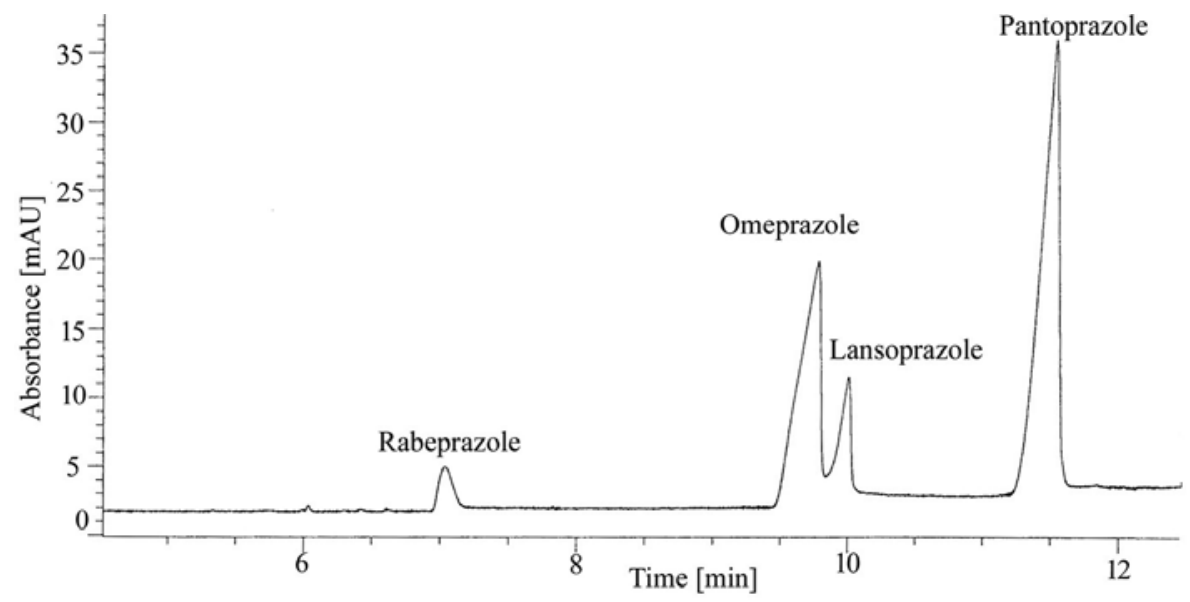

FIGURE 2 - The separation of PPIs under inital electrophoretic conditions (analytical conditions: $25 \mathrm{mM}$ phosphate buffer, $5 \mathrm{mM}$ sodium metabisulfite, $\mathrm{pH} 5.0$. temperature $20^{\circ} \mathrm{C}$, applied voltage $+20 \mathrm{kV}$, injection pressure/ time $50 \mathrm{mbar} / 2 \mathrm{sec}$., sample concentration $50 \mu \mathrm{g} \mathrm{mL} \mathrm{mL}^{-1}$, detection $300 \mathrm{~nm}$ ).

\section{Method optimization}

In order to achieve baseline separation between omeprazole and lansoprazole and a shorter analysis time ( $<10$ min.) while maintaining good resolution values between the other analytes, a face centered central composite design was applied. Based on the results of preliminary analysis, three analytical parameters were selected (BGE concentration, BGE $\mathrm{pH}$ and applied voltage); and their effect on two experimental responses (resolution value between omeprazole and lansoprazole, analysis time of the last migrating analyte) was verified.

Each experimental factor had three levels coded by $+1,0$ and -1 (Table I). Other factors, considered to have minor influence on the studied responses, were kept constant during the experiments (system temperature $20^{\circ} \mathrm{C}$, hydrodynamic injection $50 \mathrm{mbar} / 2 \mathrm{sec}$, capillary effective length $40 \mathrm{~cm}$ ). The experimental plan is summarized in Table II.

From the 20 runs performed, 6 were replicated injections in the center point of the design space (each factor being set on its medium level), 6 runs having the two factors set to medium, and one to +1 or -1 ; the remaining 8 runs represented a $2^{3}$ factorial design.

In order to evaluate the obtained experimental results, a second-order polynomial quadratic model was applied for both responses, as follows:

$$
\begin{aligned}
& \mathrm{Y}=\beta_{0}+\beta_{1} * A+\beta_{2} * B+\beta_{3} * C+\beta_{4} * A B+\beta_{5} * A C+\beta_{6} * B C+ \\
& \beta_{7} * A^{2}+\beta_{8} * B^{2}+\beta_{9} * C^{2}
\end{aligned}
$$

where $\mathrm{Y}$ represents experimental response, $\mathrm{A}, \mathrm{B}$ and $\mathrm{C}$ the experimental factors to be optimized, $\beta_{0}$ is the intercept, 
TABLE I - Levels of studied factors in the face centered central composite design

\begin{tabular}{ccccc}
\hline & Factor & \multicolumn{3}{c}{ Levels } \\
\hline $\begin{array}{c}\text { Coded } \\
\text { form }\end{array}$ & Natural form & $\mathbf{- 1}$ & $\mathbf{0}$ & $\mathbf{1}$ \\
\hline $\mathbf{A}$ & $\mathrm{pH}$ & 4.5 & 5.0 & 5.5 \\
$\mathbf{B}$ & Buffer concentration $(\mathrm{mM})$ & 25 & 37.5 & 50 \\
$\mathbf{C}$ & Voltage $(\mathrm{kV})$ & 15 & 20 & 25 \\
\hline
\end{tabular}

$\beta_{1-3}$ coefficients of linear, $\beta_{4-6}$ coefficients of interaction and $\beta_{7-9}$ coefficients of the quadratic terms.

ANOVA analysis was carried out to estimate the significance of the regression coefficients of the model. The insignificant model terms were deleted one by one, while the model was reevaluated after each deleted term.

The fitness of the model was evaluated by calculating the $R^{2}$ and $R^{2}$ adj values. $R^{2}$ expresses the fraction of the total variation explained by the model, while $\mathrm{R}^{2}$ adj is an adjusted form of $\mathrm{R}^{2}$ by considering the model coefficients to be estimated and the total number of experiments performed.
The following final regression models were obtained in coded terms; for the resolution between omeprazole and lansoprazole:

$$
\begin{aligned}
& \mathrm{R}=2.07+0.16 * \mathrm{~A}-0.33 * \mathrm{~B}+0.40 * \mathrm{C}-0.97 * \mathrm{~A}^{2}+ \\
& 0.39 * \mathrm{C}^{2}
\end{aligned}
$$

and for the migration time of pantoprazole/analysis time:

$\mathrm{t}=11.80-0.40 * \mathrm{~A}+0.56 * \mathrm{~B}-3.97 * \mathrm{C}+0.71 * \mathrm{~A}^{2}$

The calculated $R^{2}$ and $R^{2}$ adjvalues $(0.9205$ and 0.8921 for the first, 0.9768 and 0.9706 for the second model respectively) suggest that both of the estimated models have fit the experimental results.

In order to find an optimal combination of the studied analytical conditions for both measured responses, Derringer's desirability function was applied. In this approach, experimental results are transformed in desirability values on a scale between 0 and 1,0 representing the most undesirable and 1 the most desired outcome of each response of interest. In our case, resolution had to be enhanced, and analysis time had to be minimized.

TABLE II - Experimental plan of the face centered central composite design

\begin{tabular}{ccccc}
\hline Standard order & Injection order & $\begin{array}{c}\mathbf{A} \\
\mathbf{p H}\end{array}$ & $\begin{array}{c}\mathbf{B} \\
\text { (buffer concentration, mM) }\end{array}$ & $\begin{array}{c}\mathbf{C} \\
\text { (Voltage, } \mathbf{k V})\end{array}$ \\
\hline 4 & 1 & 5.5 & 50 & 15 \\
11 & 2 & 5.0 & 25 & 20 \\
1 & 3 & 4.5 & 25 & 15 \\
5 & 4 & 4.5 & 25 & 20 \\
18 & 5 & 5.0 & 37.5 & 20 \\
20 & 6 & 5.0 & 37.5 & 20 \\
17 & 7 & 5.0 & 37.5 & 20 \\
15 & 8 & 5.0 & 37.5 & 20 \\
10 & 9 & 5.5 & 37.5 & 20 \\
14 & 10 & 5.0 & 37.5 & 25 \\
9 & 11 & 4.5 & 37.5 & 20 \\
13 & 12 & 5.0 & 37.5 & 15 \\
7 & 13 & 4.5 & 50 & 25 \\
3 & 14 & 4.5 & 50 & 15 \\
2 & 15 & 5.5 & 25 & 15 \\
12 & 16 & 5.0 & 50 & 20 \\
6 & 17 & 5.5 & 25 & 25 \\
16 & 18 & 5.0 & 37.5 & 20 \\
19 & 19 & 5.0 & 37.5 & 20 \\
8 & 20 & 5.5 & 50 & 25 \\
\hline
\end{tabular}


Global desirability was calculated as geometric mean of the individual desirability values, and then the overall optimum value was searched in the experimental space.

According to the obtained regression equations, quadratic terms were included in both models, but statistically significant interaction effects were not found. All three studied factors proved to influence significantly the resolution and the analysis time as well. By increasing the $\mathrm{pH}$ (factor $\mathrm{A}$ ) of the buffer system the migration time of analytes decreased, as expected, but the resolution had an optimum $\mathrm{pH}$ value of 5.0. The analysis time increased when increasing buffer concentration (factor B), but an improvement in resolution and peak shapes was observed by increasing the ionic strength of the running buffer. Increasing the applied voltage (factor C) increased resolution and decreased analysis time. The effect of the buffer $\mathrm{pH}$ and applied voltage on resolution between omeprazole and lansoprazole, and on the analysis time of the last migrating analyte is presented in Figure 3.
A local desirability optimum was selected, based on the desirability function, for further determinations. The optimum analytical conditions consisted of $40 \mathrm{mM}$ phosphate buffer concentration (containing $5 \mathrm{mM}$ sodium metabisulfite) at $\mathrm{pH} 5.0$ and $+25 \mathrm{kV}$ applied voltage. The selected buffer concentration provided good peak shapes and resolution, which were in reasonable agreement with the increasing effect on analysis time. The $\mathrm{pH}$ value, which represented the medium level of the studied range, proved to be optimal for resolution of all analytes, while shorter analysis time was provided by the higher value of applied voltage.

Using these optimal analytical conditions good resolution values between omeprazole and lansoprazole $(\mathrm{R}=2.8, \alpha=1.03)$ and short analysis time for the last migrating analyte, pantoprazole $(8.1 \mathrm{~min}$.) were achieved. A typical electropherogram obtained from simultaneous separation of the four PPIs under the optimized conditions is presented in Figure 4. The numbers of theoretical plates
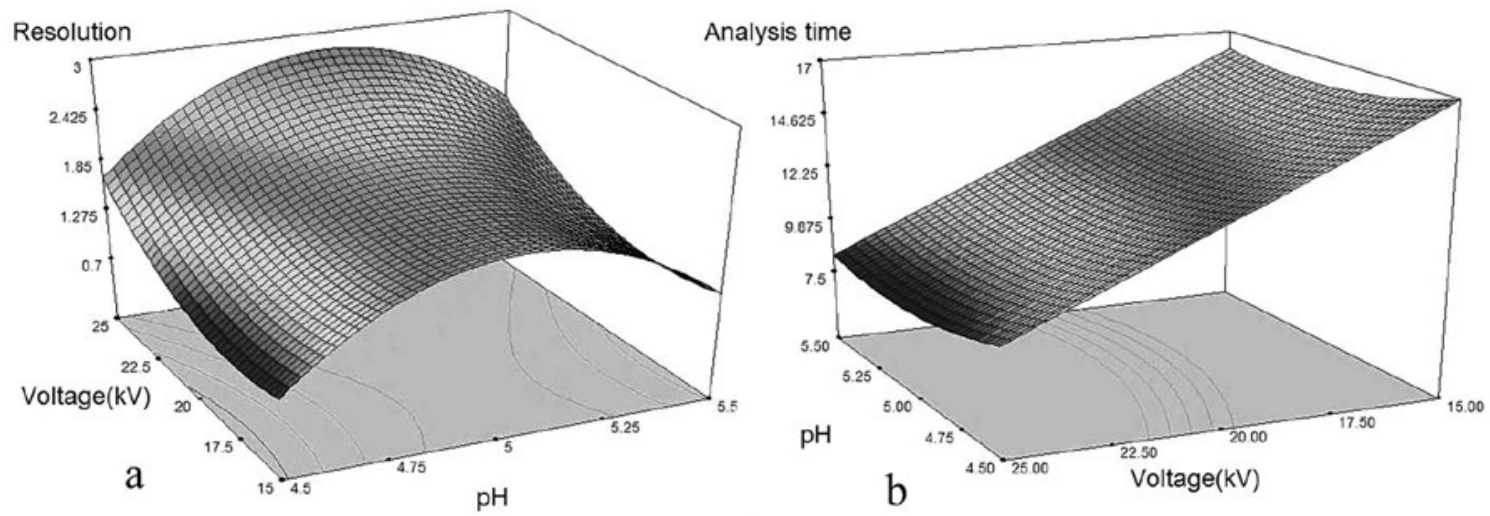

FIGURE 3 - The effects of $\mathrm{pH}$ and applied voltage on the resolution value between omeprazole and lansoprazole (a) and on the analysis time of the last migrating analyte (b) respectively.

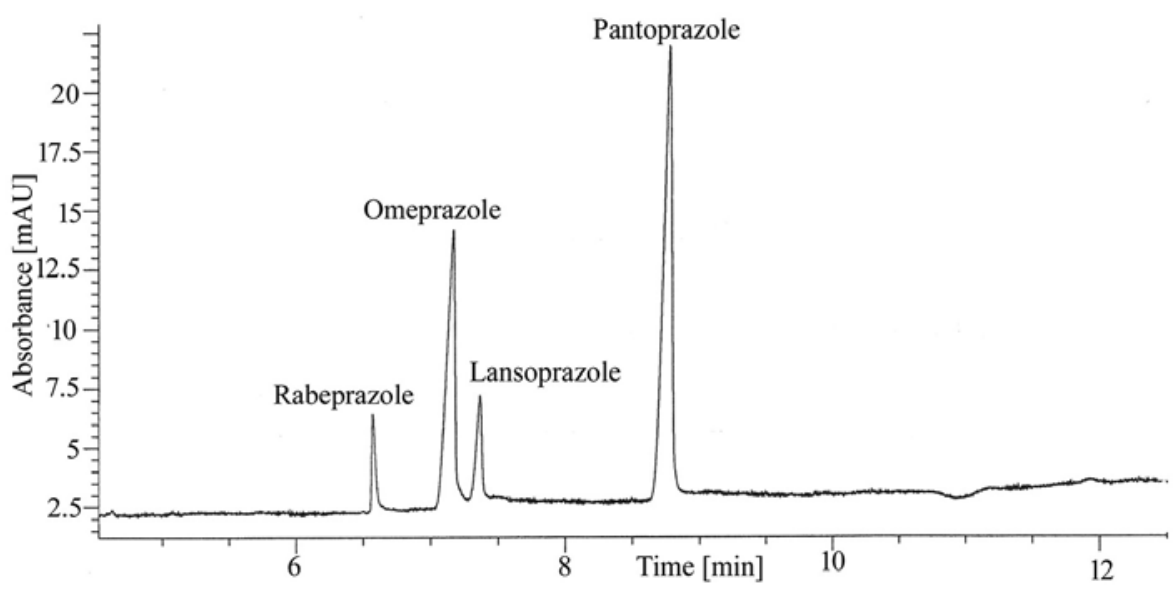

FIGURE 4 - The separation of PPIs under optimized electrophoretic conditions (analytical conditions: $40 \mathrm{mM}$ phosphate buffer, $5 \mathrm{mM}$ sodium metabisulfite, $\mathrm{pH}$ 5.0. temperature $20^{\circ} \mathrm{C}$, applied voltage $+25 \mathrm{kV}$, injection pressure/ time $50 \mathrm{mbar} / 2 \mathrm{sec}$., sample concentration $50 \mu \mathrm{g} \mathrm{mL} \mathrm{mL}^{-1}$, detection $300 \mathrm{~nm}$ ). 
Development of a generic method for the determination of proton-pump inhibitors by capillary zoneelectrophoresis

TABLE III - Regression and correlation data for the calibration curves of PPIs (concentration range $20-150 \mu \mathrm{g} \mathrm{mL} \mathrm{L}^{-1}, \mathrm{n}=6$ )

\begin{tabular}{lcccc}
\hline Analyte & Regression eqation & Correlation coefficient & LOD $(\boldsymbol{\mu g} / \mathbf{m L})$ & $\mathbf{L O Q}(\boldsymbol{\mu g} / \mathbf{m L})$ \\
\hline Rabeprazole & $\mathrm{y}=0.0663 \mathrm{x}+0.6866$ & $\mathrm{R}^{2}=0.993$ & 11.11 & 33.67 \\
Omeprazole & $\mathrm{y}=0.1986 \mathrm{x}+0.516$ & $\mathrm{R}^{2}=0.996$ & 7.88 & 23.88 \\
Lansoprazole & $\mathrm{y}=0.1581 \mathrm{x}-1.7724$ & $\mathrm{R}^{2}=0.994$ & 10.50 & 31.81 \\
Pantoprazole & $\mathrm{y}=0.2451 \mathrm{x}+1.9406$ & $\mathrm{R}^{2}=0.995$ & 5.94 & 17.99 \\
\hline
\end{tabular}

were above 130000 for all four peaks, peak symmetry factors were between 0.9 and 1.1 .

\section{Method validation}

The analytical performance of the developed method was evaluated according to ICH guidelines based on precision, accuracy, linearity, limit of detection (LOD), limit of quantification (LOQ) and robustness. For this purpose electrophoretic determinations were performed using the optimized analytical conditions on samples containing all of four studied compounds in the same concentration.

In order to investigate intra-day precision of the method, six consecutive injections $(n=6)$ were made with a standard solution $\left(\mathrm{c}=100 \mu \mathrm{g} \mathrm{mL}^{-1}\right)$ of each drug under the optimized analytical conditions. RSD values were between 0.95 and $1.25 \%$ for migration times and between 0.93 and $1.05 \%$ for peak areas. Inter-day precision was determined by performing six runs of a standard solution $\left(\mathrm{c}=100 \mu \mathrm{g} \mathrm{mL}^{-1}\right)$ under the optimized conditions over three consecutive days $(n=18)$. RSD values were between 0.78 and $1.60 \%$ for migration times and between 1.02 and $1.66 \%$ for peak areas.

Linearity of the method was investigated over the concentration range $20-150 \mu \mathrm{g} \mathrm{mL} L^{-1}$ on the basis of six measurement points and three measurements per concentration; individual linear regression equations were calculated for each PPI. Correlation coefficients were above 0.99 for all four analytes (Table III).

The limit of detection (LOD) and limit of quantification (LOQ) were estimated as standard deviation of regression equation/slope of the regression equation multiplied by 3.3 and 10, respectively (Table III).

Accuracy of the method was evaluated using recovery experiments by analyzing solutions of known concentrations within the linearity range at three levels $\left(50,100\right.$ and $\left.150 \mu \mathrm{g} \mathrm{mL}^{-1}\right)$; results of recovery study are summarized in Table IV.

The robustness of the method was demonstrated by studying the influence of the variation of several experimental parameters like buffer concentration (38-
$42 \mathrm{mM})$, applied voltage (23-27 kV) and temperature $\left(18-22^{\circ} \mathrm{C}\right)$, changing only one of these variables each time, and monitoring variation of the migration times of the analytes. Only small acceptable deviations (RSD $<2 \%$ ) were observed indicating that the robustness of the method was good.

The developed method was applied to the analysis of commercially available pharmaceutical preparations containing omeprazole. The prepared samples were analyzed in triplicate. Good agreement between the value claimed by the manufacturer and that determined by the CE method were obtained; the method presented good reproducibility (RSD $-1.17 \%$ ), and recovery was in the range $95-105 \%$ of the declared content. No interference from the drug formulation excipients could be observed on the electropherogram.

TABLE IV - Accuracy results for the determination of PPIs

\begin{tabular}{lccc}
\hline Compound & $\begin{array}{c}\text { Theoretical } \\
\text { concentration } \\
(\boldsymbol{\mu g} / \mathbf{m L})\end{array}$ & $\begin{array}{c}\text { Found } \\
\text { concentration } \\
(\boldsymbol{\mu g} / \mathbf{m L})\end{array}$ & RSD(\%) \\
\hline Rabeprazole & 50 & 49.94 & 1.63 \\
Omeprazole & & 49.16 & 0.70 \\
Lansoprazole & & 50.61 & 1.12 \\
Pantoprazole & & 49.38 & 1.10 \\
\hline Rabeprazole & 100 & 101.06 & 1.61 \\
Omeprazole & & 99.78 & 0.43 \\
Lansoprazole & & 101.37 & 1.10 \\
Pantoprazole & & 101.29 & 0.86 \\
\hline Rabeprazole & 150 & 149.12 & 0.43 \\
Omeprazole & & 149.11 & 0.38 \\
Lansoprazole & & 150.20 & 1.48 \\
Pantoprazole & & 149.56 & 0.93 \\
\hline
\end{tabular}

\section{CONCLUSIONS}

Analytical methods for determination of PPIs are usually developed individually as expected because PPIs are not used in combination during therapy. However, the 
development of a rapid analytical procedure that will not be limited to the analysis of only one PPI could be very useful.

Using a face centered central composite design, proved to be a useful approach in the optimization of the CE experiment because a large variation in experimental parameters was evaluated, gaining valuable trend information, as this broad range may not have been covered if the one factor at a time approach had been utilized. Through experimental design, it was possible to determine the experimental conditions that would lead to an optimized separation without disregarding the effects at extreme set points.

The newly developed method enables the simultaneous determination of the four studied analytes in less than 9 minutes using experimental conditions consisting of $40 \mathrm{mM}$ phosphate buffer electrolyte at $\mathrm{pH}$ $5.0,5 \mathrm{mM}$ sodium metabisulfite as antioxidant, $+25 \mathrm{kV}$ applied voltage, $20{ }^{\circ} \mathrm{C}$ system temperature, injection pressure/time $50 \mathrm{mbar} / 2 \mathrm{sec}$.

This study highlights the benefits of using universal methods for rapid quantitation of omeprazole, pantoprazole, lansoprazole and rabeprazole in a single run without the need for development of a separate and distinct method for each analyte.

\section{REFERENCES}

Bonato PS, Paias FO. Enantioselective analysis of omeprazole in pharmaceutical formulations by chiral high-performance liquid chromatography and capillary electrophoresis. J Braz Chem Soc. 2004;15(2):318-323.

Deeb SE, Wätzig H, El-Hady DA, Albishri HM, de Griend CS, Scriba GK. Recent advances in capillary electrophoretic migration techniques for pharmaceutical analysis. Electrophoresis. 2014;35(1):170-189.

Dejaegher B, Mangelings D, Vander Heyden Y. Experimental design methodologies inthe optimization of chiral CE or CEC separations: an overview. Methods Mol Biol. 2013;970:409-427.

Dogrukol-Ak D, Tuncel M, Aboul-Enein HY. The determination of lansoprazole in pharmaceutical preparation by capillary electrophoresis. Chromatographia. 2001;54(7-8):527-530.

Eberle D, Hummel RP, Kuhn R. Chiral resolution of pantoprazole sodium and related sulfoxides by complex formation with bovine serum albumin in capillary electrophoresis. J Chromatogr A. 1997;759(1-2):185-192.
El-Kommos ME, Khashaba PY, Ali HRH, El-Wekil MM. Different chromatographic and electrophoretic methods for analysis of proton pump inhibitors (PPIs): a review. J Liq Chromatogr Relat Technol. 2015;38(18):1639-1659.

Estevez P, Flor S, Boscolo O, Tripodi V, Lucangioli S. Development and validation of a capillary electrophoretic method for determination of enantiomeric purity and related substances of esomeprazole in raw materials and pellet. Electrophoresis. 2014;35(6):804-810.

Garcia CV, Sippel J, Sfair LL, Garcia SS, Jablonski A, Steppe M. Validation of a capillary electrophoresis method for analysis of rabeprazole sodium in a pharmaceutical dosage form. J AOAC Int. 2005;88(4):1081-1085.

Guan J, Yan F, Shi S, Wang S. Optimization and validation of a new CE method for the determination of pantoprazole enantiomers. Electrophoresis. 2012;33(11):1631-1636.

Guan J, Li H, Yan F, Shi S, Wang S. Optimization and validation of a novel CE method for the enantioseparation of pantoprazole and related benzimididazole using a dual chiralselector system. Electrophoresis. 2014;35(19):2800-2806.

Hancu G, Papp LA, Rusu A. Chiral separation of the enantiomers of omeprazole and pantoprazole by capillary electrophoresis. Chromatographia. 2015;78(3-4):279-284.

Lin YH, Wu SM. Analysis of omeprazole and lansoprazole in capsules by capillary zone electrophoresis. LC GC Europe. 2005;18(3):164-167.

Ma Z, Zhang L, Lin L, Ji P, Guo X. Enantioseparation of rabeprazole and omeprazole by nonaqueous capillary electrophoresis with an ephedrine-based ionic liquid as the chiral selector. Biomed Chromatogr. 2010;24(12):1332-1337.

Nevado JJB, Penalvo GC, Dorado RMR. Method development and validation for the separation and determination of omeprazole enantiomers in pharmaceutical preparation by capillary electrophoresis. Anal ChimActa. 2005;533(2):127133.

Nevado JJ, Penalvo GC, Sánchez JC Mochón MC, Dorado RM, Navarro MV. Optimization and validation of a new $\mathrm{CE}$ method for the determination of lansoprazole enantiomers in pharmaceuticals. Electrophoresis. 2009;30(16):2940-2946. 
Olsson J, Stegander F, Marlin N, Wan H, Blomberg LG. Enantiomeric separation of omeprazole and its metabolite 5 -hydroxymetabolite using non-aqueous capillary electrophoresis. J Chromatogr A. 2006;1129(2):291-295.

Olsson J, Marlin ND, Blomberg LG. Enantiomeric separation of omeperazole enantiomers by aqueous CE using UV and MS detection. Chromatographia. 2007;66(5-6):421-425.

Orlandini S, Gotti R, Furlanetto S. Multivariate optimization of capillary electrophoresismethods: a critical review. J Pharm Biomed Anal. 2014;87:290-307.
Strand DS, Kim D, Peura DA. 25 years of proton pump inhibitors: a comprehensive review. Gut Liver. 2017;11(1):2737.

Tivesten A, Folestad S, Schonbacher V, Svensson K. Nonaqueous capillary electrophoresis for the analysis of labile pharmaceutical compounds. Chromatographia. 1999;49(1):7-11.

Received for publication on $05^{\text {th }}$ December 2017 Accepted for publication on $28^{\text {th }}$ May 2018 